\section{Agroética: planteamientos críticos}

\author{
Róger Martínez Castillo*
}

\section{RESUMEN}

La preocupación ecológica constituye uno de los retos más urgentes del actual debate ético aplicado a la agricultura. Pues, la producción agrícola no solo se debe sustentar en la cantidad productivo, sino en su distribución justa, acceso a toda la población, la calidad alimentaría, estabilidad ambiental y así evitar que miles de personas a diario mueran de hambre innecesariamente. Por eso, la agroética tiene que ver más con las estructuras políticas de desarrollo agrario, el desarrollo sustentable y las necesidades de regulación, más allá de un análisis filosófico.

* Posee una Maestría y Doctorado en Agroecología por la Universidad de Córdoba, España. También tiene una Maestría en Educación Ambiental por la Universidad de Málaga, España. Es profesor Catedrático de la UCR y UNA, $\mathrm{y}$ ha escrito ponencias y ensayos sobre el tema.

Rec. $14 / 12 / 05 \quad$ Acep. 28/03/06

\section{Palabras clave}

Ética, agricultura, desarrollo, política

\section{Abstract}

Nowadays, the ecological concern is one of the most important challenges to face up to when talking about ethics and agriculture, since agricultural production should not only be referred to its productive quantity but also to its fair distribution, access to the whole population, quality, and environmental stability in order to avoid starvation. Therefore, agro-ethics is more closely related to political structures of the agrarian and sustainable development and the needs for regulations rather than to a simple philosophical analysis.

\section{Key words}

Polyphonic novel, Dostoievsky, Karamazov Brothers, Russian Literature, Realism

\section{Introducción}

A inicios del tercer milenio, el problema del hambre y la pobreza mundial se han incrementado, a pesar de que los excedentes alimenticios acumulados en los países desarrollados servirían para enfrentar decididamente este problema. Sin tomar en cuenta esta realidad, los grupos económicos que estuvieron detrás de la 
Revolución Verde (empresas de semillas y agroquímicos) argumentan, una vez más, que el problema está en el incremento de la productividad agrícola y promueven una Segunda Revolución Verde, basada en la biotecnología y en la ingeniería genética.

Pero, aun cuando se incrementaron los rendimientos de determinados cultivos, éstos se concentraron en productos de exportación y agroindustriales bajo sistemas de mono-producción a gran escala, sin un impacto significativo en la pequeña agricultura $y$, por ende, en la pobreza rural.

Sin embargo, ha llegado el momento de enfrentar socialmente el reto y la realidad de la ingeniería genética. Las compañías agroindustriales deben sentir el impacto de los movimientos ambientalistas, laborales y campesinos, de modo que reorienten su trabajo para el beneficio de toda la sociedad y de la naturaleza.

En la actualidad, la ética transversaliza las ciencias en un contexto de sociedad pluralista, secular laica, plural y transdisciplinaria, que permite un debate de los grandes temas agrarios y abre la puerta al pluralismo ético... (Marlasca, 2001).

104
Para comprender las interrelaciones éticas que existen entre la misma sociedad y su entorno natural, es necesario profundizar desde una perspectiva política. El desarrollo socio-económico y estilo de vida (producción y consumo) que juegan en este proceso.

La bioética se crea en un intento de reflexión acerca de todas las intervenciones del ser humano sobre los seres vivos, una reflexión difícil de alcanzar ya que su objetivo principal es la de identificar valores y normas que guíen el actuar humano, la intervención de la ciencia y de la tecnología en la vida misma y en la biosfera.

Es poco cuestionable decir que las tecnologías no son buenas ni malas, que lo único que importa es quiénes son los usuarios y para qué las usan: al mercado. Tal argumentación, no esclarece para nada el rol de la ciencia y la tecnología en la sociedad ni el lugar del científico en relación con la estructura de poder.

Según este argumento, el quehacer científico y el desarrollo tecnológico son objetivos y neutrales y persiguen fines universalmente positivos. Si es así, entonces no deben ser cuestionados nunca. Ergo, toda crítica a la ciencia y tecnología es inoportuna e indebida, fuera 
de su contexto de interrelación. El argumento de la neutralidad le permite al "trabajador científico o empresario" desentenderse de toda responsabilidad por los impactos negativos que tenga su labor. Si un trabajo de investigación y/o desarrollo resulta en graves descalabros sociales o ecológicos, eso es responsabilidad y culpa de otros, o bien, si la actividad se da por aumentar las ganancias, sin tomar en consideración sus consecuencias socio-ambientales.

Asumir que la naturaleza es sujeto de valor implica plantear una perspectiva anti-ética mercadocéntrica; por eso, la bioética se dedica a los siguientes aspectos:

1. investiga los problemas éticos de las profesiones sociales y naturales;

2. estudia problemas éticos en investigaciones sobre el ser humano y la sociedad;

3. atiende a los problemas sociales inherentes a las políticas sanitarias, medicina del trabajo y a políticas de control natal;

4. analiza problemas relacionados con la intervención de la vida de los demás seres vivos $y$ el equilibrio del ecosistema;
5. investiga la aplicación de la bioética en lo forense: cuerpo humano y valores.

La paradoja que encierra el mercado con su pretensión de hacer posible la vida humana, es que niega el principio mismo que la sustenta y enfatiza en extraer sus ganancias, a la vez que pretende abastecer a la humanidad de sus necesidades prioritarias, le quita a la vez la fuente de su existencia: la tierra misma, su ecosistema. Por un lado estos principios traen aparejada la victimización de los sujetos a los cuales debía beneficiar, y por otro lado, convierten al ecosistema en un campo devastado ya que él representa la fuente primaria de donde la industria produce sus ganancias.

La bioética lucha, por una parte, por la dignidad e integridad de todo la persona humana, y por otra descubre que la salud no es privilegio, sino un derecho humano universal (...) porque estamos viendo cómo la misma ciencia y la tecnología que la acompañan hacen peligrar hasta la supervivencia de nuestra especie" (Trevijano, 1999:81). Y dentro de este marco de conocimiento se debe partir hacia la conceptualización de una ética ambiental ajena a cualquier discurso tergiversado, como el de 
la economía política liberal o de las modernas ciencias, algunas de ellas no honestas con la objetividad y responsabilidad que le deben a los demás seres vivientes.

Se trata de cuestionar las falsas promesas hechas por la agroindustria (transgénicos), al prometer que los cultivos producidos mediante una tecnología, que generarían una agricultura menos dependiente en insumos químicos, aumentarían la productividad y ayudarían a reducir los problemas ambientales. Pero, que sus efectos sobre las condiciones sociales y económicas, y los valores culturales, religiosos y morales de las diferentes culturas, que han sido ignorados reiteradamente en el desarrollo tecnológico.

\section{Ética y ambiente}

La bioética, como parte básica del saber ético, resuelve los problemas de la moral práctica, dentro del quehacer científico y donde está implicada la vida humana y su entorno. ¿Qué es tan importante para la vida del hombre? Las demás especies, sean estas animales o vegetales. La bioética es de gran importancia para el análisis de la situación científica en la actualidad, pues en ella confluye todo el conocimiento del hombre, siendo un punto de unión entre las ciencias humanas (Restrepo, 2000).

Mediante el discurso ético se pretende la conservación del ser humano y del ecosistema que lo rodea la naturaleza. Así aparece el discurso de la ética ambiental, ecológica, planetaria. De tal manera, "...la ética ecológica aporta una filosofía de la conservación y de la garantía prioritaria de los bienes ecológicos que es muy fructifica para las precauciones que tratan de establecer los principios de la bioética" (Garrido,2002:246). La bioética, dado que es una disciplina multidisciplinar, viene a ser el marco de referencia de la actual reflexión ética sobre el ambiente natural y social.

Se parte de una serie de supuestos que se creen están avalados por la experiencia y no hay en ellos nada contrario a la razón práctica:

a) Todo organismo vivo en condiciones habituales de elección tenderá a preferir la duración a la destrucción.

b) Todo organismo vivo, en condiciones habituales, tenderá a reforzar aquellas situaciones que le procuren sensaciones no dolorosas. 
c) Todo organismo vivo tenderá a reforzar aquellas condiciones que le garanticen su supervivencia y comodidad.

d) Todo organismo vivo tiene un compromiso con la supervivencia no solo de su individualidad sino de la especie y los ecosistemas en los que esta se reproduce. Hay un vínculo con las formas de vida que le precedieron y con las que le continuarán.

e) Todo organismo vivo tiende a elegir aquellas conductas, que sin poner en peligro su individualidad, garanticen las condiciones de reproducción de la especie.

f) Todo organismo vivo individual se vincula con su sistema y ecosistema a través de la forma y se diferencia de su entorno por medio de la forma. Luego es la forma la que le da continuidad, especialidad, universalidad, comunicación; es la forma la que garantiza, por encima de las contingencias y la arbitrariedad del azar, la persistencia de la identidad y la producción de la diferencia.

g) Todo organismo vivo busca la adecuación formal, lo cual supone la adecuación tempo- ral y estructural en su propia esencia vital. La orientación hacia la comodidad ontológica es una orientación rectora de la conducta de los seres vivos. La proliferación y el derroche en los seres vivos sirven a la comodidad y a la garantía de la duración. La complejidad es un resultante de esta tensión entre derroche y eficacia.

En la tecnología y derechos humanos, la ingeniería genética se plantea como el salto de la humanidad hacia fuera de sí; entre sus supuestos están los siguientes:

- el desarrollo tecno-científico está cada vez más implicado en la problemática planetaria, civilizatoria, societaria, humana.

- los seres humanos son quienes llevan adelante las diversas e inevitables transformaciones, porque es precisamente nuestra condición humana, nuestra forma de estar en el mundo. $Y$ son esas transformaciones las que tienen que estar cada vez más bajo la lupa por sus implicancias cada vez más abarcativas y que patentiza que "todos estamos en el mismo barco".

- la ingeniería genética constituye un acontecimiento militar 
dentro de este cambiante universo de transformaciones en que se degrada la vida.

Si se aplican estos criterios axiomáticos a la supuesta obligación ética de garantizar unas condiciones de vida mínimamente dignas para las generaciones futuras, esto tendría consecuencias claramente definidas en los procedimientos y principios éticos y políticos. De poder interpretar las exigencias que serían deseables por las generaciones futuras a la luz de estos criterios se tendrían que convenir que:

- $\quad$ recibir condiciones de vida que le garanticen la supervivencia.

- $\quad$ recibir condiciones naturales más cómodas posibles.

- recibir un patrimonio natural mayor y mejor conservado.

- muestra abstracta de lo esencial tiene consecuencias en lo concreto.

De ese conjunto de preferencias se derivan la obligatoriedad ética y la preferencia por los valores, principios, derechos y normas que estén en coherencia con estos presupuestos. Así se deduce la opción por el "principio ecológico de con- servación" en coherencia directa con el "interés inter-subjetivo y ecológico de la especie".

Otro argumento favorable a la idoneidad del principio de "preferencia de la conservación", en el cumplimiento de las obligaciones morales de solidaridad ontológica con las generaciones pasadas y futuras. Este argumento es el de la irreversibilidad. Las estrategias derivadas del principio de "preferencia de la conservación" no secuestran la libertad de las generaciones futuras: siempre es posible destruir aquello que ha sido legado; siempre es posible hacer desaparecer especies o bienes renovables, o empobrecer la biodiversidad. Pero si se legan especies y ecosistemas extinguidos, recursos no renovables agotados o un grado de biodiversidad escaso: estará secuestrada la libertad y capacidad de decisión de las generaciones futuras.

Presuponer, que las generaciones futuras decidirían que nuestro comportamiento en relación con sus deseos, tal como se presupone aquí que se harían; es una suposición contra-fáctica que favorece su libertad de decisión:

- supervivencia de las condiciones de posibilidad de la vida. 
- continuidad ininterrumpida de la libertad: que ninguna decisión libre secuestre a la libertad del futuro, que nunca la libertad concreta destruya a la libertad abstracta. Que el ejercicio de la libertad en una situación determinada, o en una generación concreta, no destruya a la libertad de las venideras.

En estas dos constantes se preservan la continuidad (duración) de la forma de la vida y de la forma del poder constituyente. La continuidad que nos une y nos separa de todo lo que hay, y de nuestra propia constitución como especie política. Las obligaciones morales y políticas con las generaciones futuras son la base sobre la que se asienta la democracia como forma de vida.

La opción por un sistema ético centrado en la ética pública y por tanto compuesto de valores, principios y normas formales, procesales, pluralistas y relativistas supone conectar la argumentación legitimadora con la argumentación explicativa.

El problema que se plantea no es elegir entre crecimiento y calidad del ambiente natural, sino tratar de compatibilizar objetivos socio-económicos y ambientales, mediante modelos de manejo de bienes y métodos de crecimiento (Sachs, 1998).

\section{Biotecnología y hambre}

Los defensores de la Revolución Verde sostienen que los países de América Latina deberían optar por un modelo agroindustrial basado en variedades mejoradas y en el creciente uso de fertilizantes y pesticidas, a fin de proporcionar una provisión adicional de alimentos a sus crecientes poblaciones y economías. El problema es que la biotecnología no reduce el uso de agroquímicos ni aumenta los rendimientos. Tampoco beneficia a los consumidores ni a los agricultores pobres. Dado este escenario, un creciente número de agricultores, ONGs y otros propulsores de la agricultura sustentable proponen que, en lugar de este enfoque intensivo en capital e insumos, los países de la región deberían propiciar un modelo agroecológico que da énfasis a la biodiversidad, el reciclaje de nutrientes, la sinergia entre cultivos, animales, suelos y otros componentes biológicos, así como a la regeneración y conservación de los recursos (Altieri, 2000). 
Sin embargo, las compañías de biotecnología sostienen que los organismos genéticamente modificados son hallazgos científicos necesarios para alimentar al mundo y reducir la pobreza en los países en desarrollo. La mayoría de las organizaciones internacionales encargadas de la política y de la investigación para el mejoramiento de la seguridad alimentaria en el mundo en desarrollo y los gobiernos y hacen eco de este enfoque, que se basa en dos suposiciones críticas: que el hambre se debe a una brecha entre la producción de alimentos y la densidad de la población humana o la tasa de crecimiento; y que la ingeniería genética es la única o la mejor forma de incrementar la producción agrícola y por lo tanto de cubrir las futuras necesidades de alimento.

Un punto inicial para aclarar estas falsas concepciones es entender que no hay una relación entre la presencia del hambre en un país determinado y su población. Pues, el mundo produce hoy más alimentos, tanto que una persona puede comer 14 platos de comida al día (FAO,2002). Hay suficiente alimento disponible para proveer 4,3 libras por persona cada día; 2,5 libras de granos, frijoles y nueces; alrededor de una libra de carne, leche y huevos y otra de frutas y verduras (Lappé FM, et al.1998).

En 1999 se produjo suficiente cantidad de granos en el mundo para alimentar una población de ocho mil millones de personas (seis mil millones habitaron el planeta en el 2000), si éstos se distribuyeran equitativamente $\mathrm{o}$ no se dieran como alimento a los animales. Canalizando un tercio de los granos producidos en el mundo hacia la población hambrienta y necesitada, el hambre cesaría instantáneamente.

El hambre también ha sido creada por la globalización, especialmente cuando los países en desarrollo adoptan las políticas de libre comercio exigidas por agencias internacionales (reduciendo los aranceles y permitiendo el flujo de productos de los países industrializados). La experiencia de Haití, uno de los países más pobres del mundo, es ilustrativa. En 1986 Haití importó sólo 7000 toneladas de arroz, porque la mayor parte se producía en la isla. Cuando abrió su economía al mundo, los inundó un arroz más barato proveniente de los Estados Unidos, donde la industria del arroz es subsidiada. En 1996, Haití importó 196000 tone- 
ladas de arroz al costo de US\$ 100 millones. La producción de arroz haitiano se volvió insignificante cuando se concretó la dependencia en el arroz extranjero. El hambre se incrementó (Aristide JB, 2000).

Las causas reales del hambre son la pobreza, la desigualdad y la falta de acceso a los alimentos y a la tierra. Demasiada gente es muy pobre (alrededor de dos mil millones sobreviven con menos de un dólar al día) para comprar los alimentos disponibles (a menudo con una pobre distribución) o carecen de tierra y los recursos para sembrarla (Lappé FM, et al.1998). Porque la verdadera raíz del hambre es la desigualdad, cualquier método diseñado para reforzar la producción de alimentos, pero que agudice esta desigualdad, fracasará en reducir el hambre. Por el contrario, sólo las tecnologías que tengan efectos positivos en la distribución de la riqueza, el ingreso y los activos, que estén a favor de los pobres, podrán en realidad reducir el hambre. Afortunadamente, tales tecnologías existen y pueden agruparse bajo la disciplina de la agroecología (Altieri $M A$, Rosset P, Thrupp LA,1998; Uphoff N, Altieri MA.,1998).
Atacando la desigualdad por medio de reformas agrarias se mantiene la promesa de un aumento de la productividad que sobrepasa el potencial de la biotecnología agrícola. Mientras que los defensores de la industria hacen una promesa de 15 - 20 e incluso $30 \%$ de aumento de los rendimientos por la biotecnología, los pequeños agricultores producen hoy de 200 a $1000 \%$ más por unidad de área que las grandes fincas a nivel mundial (Rosset P.;1999). Una estrategia clara para tomar ventaja de la productividad de las pequeñas fincas es impulsar reformas agrarias que reduzcan las grandes propiedades ineficientes e improductivas a un tamaño pequeño óptimo $y$, así, proporcionar las bases para el incremento de la producción en fincas de pequeños agricultores, incrementos ante los cuales se diluiría la publicitada promesa productiva de la biotecnología.

Es importante entender que la mayor parte de innovaciones en la biotecnología agrícola se orientan a las ganancias privadas más que a las necesidades sociales. El verdadero motor de la industria de la ingeniería genética no es hacer la agricultura más productiva, sino generar mayores ingresos (Busch $L$, et al.; 1990). 
Esto se evidencia revisando las principales tecnologías del mercado de hoy:

i. cultivos resistentes a los herbicidas, como la Soya Ready Roundup de Monsanto, semillas que son tolerantes al herbicida Roundup de Monsanto, y

ii. los cultivos Bt (Bacillus thuringiensis) que han sido desarrollados por ingeniería genética para producir su propio insecticida. Primero, la meta es ganar más participación del mercado de los herbicidas para un producto exclusivo $y$, en el segundo, aumentar las ventas de semillas aun a costa de dañar la utilidad de un producto clave para el manejo de plagas (el insecticida microbiano a base de $\mathrm{Bt}$ ) en el que confían muchos agricultores.

Estas tecnologías responden a la necesidad de las compañías de biotecnología de intensificar la dependencia de los agricultores de semillas protegidas por la llamada "propiedad intelectual", que entra en conflicto directamente con los antiguos derechos de los agricultores a reproducir, compartir o almacenar semillas (Fowler C, Mooney P; 1990). Las corporaciones obligan a los agricultores a comprar una marca de insumos de la compañía y les prohíben guardar o vender la semilla, controlando el germoplasma, desde la producción de semillas hasta su venta, y obligando a los agricultores a pagar precios inflados por paquetes de semillas químicos, por lo que las compañías extraen el máximo beneficio de su inversión (Krimsky S, Wrubel $R P ., 1996)$.

\section{Cuestionamiento ético de la biotecnología}

Al cuestionar los mitos de la biotecnología, se evidencia que la ingeniería genética es realmente otra ronda tecnológica o "varita mágica" destinada a entrampar los problemas ambientales de la agricultura sin cuestionar las raíces causales que ocasionaron los problemas la primera vez (Hindmarsh, 1991:21). La biotecnología desarrolla soluciones monogénicas, diseñadas sobre modelos industriales de eficiencia, para problemas que derivan de sistemas de monocultivo ecológicamente inestables. Este enfoque unilateral y reduccionista, que utilizó el paradigma de "un químico una plaga", y que llevó a problemas de resistencias de plagas y contaminación ambiental, 
no difiere del biotecnológico que aplica "un gen una plaga" y lleva a las mismas falacias ecológicas.

La agricultura industrial moderna, hoy caracterizada por el modelo de la biotecnología, se basa en una premisa filosófica que es fundamentalmente errónea y que necesita ser expuesta y criticada para avanzar hacia una agricultura verdaderamente sustentable. Esto es muy relevante en el caso de la biotecnología, donde la alianza de la ciencia reduccionista y la industria multinacional monopolizadora lleva a la agricultura por un camino equivocado. La biotecnología percibe los problemas agrícolas como deficiencias genéticas de los organismos y trata a la naturaleza como una mercancía y, en el camino, hace a los agricultores más dependientes de un sector de agronegocios que concentra cada vez más su poder sobre el sistema alimentario. Es preocupante, que las motivaciones económicas, más que las preocupaciones sociales y ambientales, sean las que determinan el tipo de investigación y las modalidades de producción agrícola que prevalecen en todo el mundo (Busch, L, et al. 1990).

Las críticas ambientalistas a la biotecnología cuestionan las suposiciones de que la ciencia de la bio- tecnología está libre de valores y que no puede estar equivocada o ser mal utilizada, y piden una evaluación ética de la investigación en ingeniería genética y sus productos (Krimsky S, Wrubel RP. 1996). Se considera que quienes promueven la biotecnología tienen una visión utilitaria de la naturaleza y favorecen el libre intercambio (tradeoff) de las ganancias económicas por el daño ecológico, manteniéndose indiferentes ante las consecuencias que acarrean para los seres humanos. En el corazón de la crítica están los efectos biotecnológicos sobre las condiciones sociales y económicas y los valores culturales, religiosos y morales que se han ignorado. Todo esto ha llevado a una serie de científicos y filósofos a plantearse una serie de preguntas que abordan temas tales como:

- Propósitos de la tecnología: ¿Cuál es la real necesidad de esta tecnología? ¿A qué necesidades responde la biotecnología? ¿Quién dice qué se necesita y por qué? ¿Qué problemas va a solucionar? ¿Cuál es la causa de estos problemas y acaso la biotecnología realmente los enfrenta de raíz? ¿Cómo afecta la tecnología a lo que se está produciendo? ¿Cómo, para qué y para quién se está produciendo? ¿Cuáles 
son las metas sociales y criterios éticos que guían la investigación biotecnológica?

- Definiciones de riesgo: ¿Se han considerado los efectos ambientales, sociales, económicos y políticos? ¿Quién ha definido el alcance y significancia de estos efectos? ¿Son los efectos irreversibles? ¿Se están midiendo estos efectos en comparación con tecnologías más blandas existentes? ¿Cómo se determina la aceptabilidad del riesgo y quién lo hace? ¿Acaso la biotecnología cierra opciones para el futuro? ¿O se promueve a expensas de otros enfoques? ¿Cuáles han sido las alternativas ignoradas? ¿Cómo se estiman los beneficios?

- Distribución de riesgos y beneficios: ¿Cómo se distribuyen los costos y beneficios? ¿Quién se beneficia de la biotecnología? ¿Quién pierde? ¿Cómo se determina, y por quién, que la distribución es aceptable? ¿Acaso la biotecnología concentra poder y en manos de quién?

- Alternativas: ¿Se han explorado a fondo alternativas para solucionar problemas que la biotecnología dice solucionar? ¿Esta exploración examina una serie de intereses e incluye la participación de todos los grupos sociales? ¿Está el proceso abierto al escrutinio público? ¿Se distribuyen equitativamente los fondos de investigación para las alternativas? ¿Existen alternativas ambientalmente menos nocivas y socialmente más justas?

\section{Agroética}

La preocupación agrícola desde la perspectiva ecológica constituye uno de los retos más urgentes del debate ético. La sociedad empieza a reconocer que está entrelazada con el ambiente que depende de él y que no se debe desligar sin sufrir daños. Este nuevo paradigma se resume como la "conciencia ecológica" (Gafo, 2001).

La agroética es una concepción social, política de las relaciones humanas hacia el ambiente y la misma sociedad. Regula la acción humana (economía, producción) hacia el ambiente. Ciencia y tecnología: al servicio de la humanidad, no de pequeños sectores dominantes y su visión mercantil, para mitigar problemas socio-ambientales. 
El objetivo de la agroética es muy amplio, ya que tiene que ver con la conducta y relaciones humanas. Entre los temas de estudio sobre el ambiente están los problemas generados por las modernas tecnologías en el hábitat natural: ecología y ética ambiental, relaciones humanas, destrucción de ciertas especies y otros.

El desarrollo insustentable no es resultado de una mala planificación o ejecución de un proyecto, ni falta de visión o mala estructuración institucional. La crisis socio-ambiental no es tampoco, un problema técnico, ni tecnológico, ni económico, ni social; es ético, moral, es político, con sus estructuras de relaciones deshumanizadas y desnaturalizadas, que enfatizan en mercados desregularizados. El desarrollo dominante no comparte de modo equitativo, ni toma en cuenta las necesidades locales, para eliminar la pobreza y el hambre debidamente. Este enfatiza en maximizar la producción y consumo para cubrir sus intereses, en detrimento de necesidades y responsabilidades socio-ambientales.

Para alimentar el mundo, combatir la pobreza y proteger el ambiente existe toda una gama de tecnologías y técnicas ecológicas, que muestran gran promesa y reali- zarían su pleno potencial si sólo tuvieran el apoyo y financiamiento necesarios.

Estas áreas de investigación requieren de gran rigor metodológico y extensa experimentación, por lo que no se puede decir que los ecologistas estemos opuestos al cambio y la tecnología. Pero tales opciones tecnológicas van en contra de los grandes intereses que son los que precisamente controlan y financian gran parte de la investigación científica. Por lo tanto, el adelantarlas requiere de una estrategia política alternativa, de corte antiimperialista y antineoliberal.

\section{Bioseguridad}

Se refiere al conjunto de normas jurídicas y políticas corporativas, estatales y/o supranacionales adoptadas para garantizar la segura aplicación de la biotecnología en la salud humana y conservación del ambiente. La bioseguridad nace como una necesidad en los países que desarrollaron programas de biotecnología, sin embargo la bioseguridad es esencial para los países que luchan por un desarrollo que no comprometa la supervivencia de generaciones futuras; así, los países en vías 
de expansión han desarrollado diversas legislaciones en el ámbito nacional y supranacional.

La tecnología de la vida ha producido tantas interrogantes de orden ético, que ya se habla de una nueva disciplina, la bioética.

La ética es parte de la filosofía que trata de la moral y de las obligaciones humanas, en cuanto se relacionan con los fines que determinan su rectitud, sus diversas nociones relativas al bien y el mal y de cómo éstas se relacionan con sus semejantes y con la diversidad de formas de vida que les rodea. La bioética es la disciplina que estudia el carácter ético de las técnicas aplicadas sobre los seres vivos.

Pero, la pregunta clave es: ¿a quién le sirve la tecnología o el capital?

En la ética, la "crisis ambiental" se basa en cómo el ser humano se ha relacionado consigo mismo y con el ambiente; sin embargo, conociendo las causas de su deterioro, ha mantenido y mantiene la postura irresponsable, como el mercado desgularizado.

La agroética es el estudio sistémico de la conducta humana en el manejo de los bienes naturales, mediante las ciencias de la vida y cuidado de la salud, en cuanto dicha conducta es examinada a la luz de los valores y principios morales y la responsabilidad que respalda la crítica.

No es la razón, sino el sentimiento lo que establece el primer contacto con la realidad, y la "gran crisis actual no es económica, política o religiosa y sí una crisis de afectividad, de la capacidad de sentirse vinculado al otro. Afectividad, cuidado, cooperación y responsabilidad son los cuatro principios centrales de una "ética salvadora" que la humanidad debe adoptar con urgencia para evitar su propia extinción.

Es el principio de la responsabilidad lo que respalda la crítica a los productos transgénicos, la necesidad de precaución ante las consecuencias desconocidas, la posibilidad de que la modificación genética de un alimento pueda romper el equilibrio entre los "miles de millones de bacterias" y moléculas que componen un ser humano.

La bioética debe ser una concepción social, política de las relaciones humanas hacia el ambiente natural y la misma sociedad; regular la acción humana (economía, producción) hacia el ambiente. Ciencia y tecnología: al servicio de la humanidad, no de pequeños sectores dominantes y su visión 
mercantil. Mercado comercialregulado para mitigar problemas socio-ambientales.

El objetivo de la bioética es amplio, ya que tiene que ver con la conducta humana, donde estudiaría la ética de la biología, medicina, antropología, sociología y otros.

La crisis socio-ambiental es efecto de la degradación de la naturaleza en beneficio privado, donde los intereses corporativos ponen en peligro la producción y reproducción de la vida humana y todos los seres vivientes. El abuso de la economía desregularizada sobre los bienes naturales (árboles, agua, minerales), ofende y deteriora a la madre tierra.

La agroética es una nueva tendencia teórica, que aborda los problemas éticos de la ciencia y la tecnología moderna en relación con la temática agraria. La sociedad no es homogénea ni unitaria por lo que se debe reconocer dicha realidad (Carvajal, 2003).

De esta forma, se justifica que uno de los temas más importantes tratados por la agroética: el ambiente y sociedad, sean el eje; partiendo del hecho de que los problemas socio-ambientales se han agravado conforme avanza el tiempo y los conocimientos humanos, que no siempre han traído beneficios. Esto debido al abuso de tales "herramientas para una mejor calidad de vida humana y desarrollo".

La agroética reflexiona acerca de la intervención del ser humano sobre los demás seres vivos: una reflexión difícil. Pero la idea es contextualizarla en sus relaciones políticas, económicas e interacción con su entorno natural.

La agroética adquiere importancia a raíz de los problemas políticos que se suscitan la generación, transferencia y aplicación de avances de las ciencias y tecnologías agrarias. El enfoque sustentable responde a una historia que ha valorado al ambiente en función de la utilidad para las necesidades del ser humano.

Es cuestionable decir que las tecnologías no son buenas ni malas, que lo único que importa es quiénes son los usuarios y para qué las usan, pues, no aclara el papel de la ciencia y tecnología en la sociedad, ni el lugar del científico en relación con la estructura e intereses de poder.

La encrucijada social y ecológica que enfrenta la sociedad y el mundo requiere que el científico, informado por los conceptos de desarrollo sustentable y principio 
precautorio, cuestione e indague quién dirige el desarrollo de la ciencia, quién la financia, respondiendo a qué intereses y con cuales propósitos.

La reflexión sobre el desarrollo sustentable surge del interés de abordar la problemática ecológica con las circunstancias, condiciones, estilos y políticas de desarrollo social, económico, político y cultural. La agroética se aplica al desarrollo sustentable como:

- respeto y preservación de las relaciones de sustentabilidad.

- aseguramiento de la salud de productores y consumidores.

- distribución justa y equitativa de beneficios.

- justicia intergeneracional: no comprometer el bienestar de futuras generaciones.

La bioética, al incluir los procesos vitales y orgánicos, vida social, ambiental o biosfera, se examina mediante deberes, derechos, obligaciones morales que permiten:

- conservar, proteger, mejorar y no dañar la calidad de vida y del ambiente.

- respetar el derecho y capacidad de autogobierno y competencia de seres humanos o comunidades a decisiones que tienen que ver con las predilecciones y demandas biológicas (alimentarías, sanitarias, reproductivas, sexuales, ambientales).

- acceso y distribución justa de servicios y satisfacción de demandas biológicas.

El debate sobre los problemas de la agroética en el mundo globalizado de nuestros días pasa necesariamente por reconocer la confrontación entre dos grandes modelos de desarrollo rural, uno de inspiración neoliberal y otro basado en el desarrollo sustentable, fundamentado en la soberanía alimentaría.

Los problemas socio-ambientales son temas importantes de estudio en los organismos internacionales, instituciones nacionales y locales. Problemas, como el crecimiento global de la población mundial y su relación con los (agro)ecosistemas, la deforestación, lluvia ácida, capa de ozono, efecto de invernadero, son preocupación de la humanidad, que pone en peligro la continuidad de la especie humana sobre la tierra. Sin embargo, no se toma una conciencia política de que es el propio modelo (estilo) de desarrollo dominante, el marco en el que se generan dichos desequilibrios socio-ambientales. 
El sistema económico-productivo vigente, con sus políticas desarrollistas neoliberales y mercadocéntricas agravan estos problemas. De ahí que la agroética debe cumplir con la necesidad de un cambio estructural ante la crisis del desarrollo irracional.

La agroética analiza la estructura socio-económica y política de los seres humanos en tanto que afectan a los ecosistemas y la misma sociedad. Por eso, la agroética no es neutra. Nada en este mundo es neutral, ni siquiera la ciencia y tecnología o el capital, pues son procesos políticos y nunca se realizan en aislamiento de intereses económicos y estructuras de poder (Beck, 1998).

La dominación interna / externa impide el surgimiento de toda conciencia crítica. Esta requiere de alternativas comprometidas, que rompan con la dependencia y se preocupe de las necesidades colectivas y nacionales. Por eso, debe estar orientada hacia una praxis política crítica que promueva la transformación social y ambiental.

El conflicto entre la conservación natural y el usufructo humano no tiene solución bajo el modelo societario convencional. Solo una nueva perspectiva basada en la alianza estratégica entre la naturaleza (diversidad, que potencie un desarrollo alternativo) y un desarrollo real sustentable hará posible esta realidad. Revalorizar la importancia del conocimiento histórico ambiental es una prueba a la tolerancia, a la diversidad y enriquecimiento de la cultura nacional.

Ante los nuevos paradigmas, la agroética politizada constituye una alternativa dirigida a lograr una acción educativa, que convierta al individuo en sujeto de su propio destino, comprometido con su sociedad y conciencia de los problemas socio-ambientales, buscando soluciones necesarias para un desarrollo ambiental sustentable.

La agroética política plantea un nuevo desarrollo socio-económico alternativo, un cambio estructural radical, toda una revolución, donde lo ambiental sea eje, mediante la autogestión de las comunidades locales, participación activa, descentralización, democratización y transformación de las relaciones de poder, donde el entorno predominante nacional (institucional) e internacional (financiero) y la economía de mercado regularizada tome en cuenta lo natural y social. 
En la medida en que los agricultores apliquen la agroética de manera holística y analicen críticamente los problemas, aciertos y necesidades actuales, en esa medida estarán proponiendo planes de manejo sustentable del agroecosistema que aseguren la participación de la población a una existencia digna.

La ética ambiental está llamada a ser tributaria de esta opción, la liberación para la vida plena, con abundancia de recursos de vida, lo que demanda modificar las relaciones sociales y hacerlas más justas. Ello exige una opción de carácter más radical: la opción por la vida, unida a la justicia y la paz, y a la par de ello está el problema del ambiente. Se trata de lograr un nuevo estilo de vida que haga consciente al ser humano de que él es parte integrante del ambiente, independientemente de las diferencias ideológicas, étnicas y culturales.

Es necesaria una ética ambiental en términos globales. No se trata de relaciones anónimas, ni de relaciones funcionales con intereses mezquinos, sino de relaciones integrales, cuyo punto de referencia sea la responsabilidad ante la vida e historia.

Se debe plantear si la reconstrucción de la sociedad va a ser alre- dedor del individualismo egoísta que nos plantea el neoliberalismo, o a través de la solidaridad, el reconocimiento y el respeto del otro, si se va a ser a través de la competencia, cuyas reglas de juego no importan más que el resultado que se logre, o si se va a efectuarse mediante la cooperación del pensamiento colectivo e intercambio de saberes.

Los desafíos más importantes que debe enfrentar todo científico-profesional tomar partido, estar comprometido con la sociedad misma y el ecosistema, no con el mercado desregularizado y neoliberal.

La crisis de valores, la perdida de sentido, la ruptura de los lazos solidarios y la búsqueda del mayor rédito a cualquier costo son algunas de las principales preocupaciones que inundan el arco social, determinando las conductas humanas.

Paralelo, y en función de la reconversión del mercado laboral, muchas carreras han modificado sus planes de estudio para responder al debate acerca del perfil del graduado que egresa de universidades. Así, no solamente la adaptación del profesional al mundo del trabajo está en discusión, sino también la formación ética del mismo, al punto de que más allá 
de los conocimientos específicos y técnicos de su profesión, la propia universidad se ve en la necesidad de generar espacios como lo establecen los principios de "adquirir, conservar, acrecentar y transmitir críticamente el conocimiento, orientando su accionar a la formación plena de profesionales con compromiso social y sentido de la ética socio-ambiental.

Es necesario que el Estado intervenga para que haya una planificación y organización en función de determinados objetivos, y no solo esfuerzos individuales $y$, que él mismo tenga el suficiente poder como para hacer cumplir las leyes que genera, sin que sus funcionarios se dejen amedrentar, comprar o cooptar por otros grupos de poder.

Mientras los Estados se debilitan y crecen las fuerzas de las multinacionales, disminuyen proporcionalmente las posibilidades de que éstos promuevan leyes que puedan perjudicar los intereses de las grandes empresas o hacer que las existentes sean cumplidas. Esta es una de las razones por las que se cree que los ecologistas deben intervenir en política, aunque no significa enrolarse en un partido político.
Son importantes las decisiones que se adopten respecto a la forma de vida, como aplicar las cuatro R (Reduce, Reutiliza, Recicla, Rechazar), y el consumir local, ahorro de energía o agua; pero eso solo no alcanza para lograr que la sociedad futura viva en un mundo mejor del que se les está dejando.

\section{Agroecología: alternativa real}

Una estrategia de desarrollo agrícola sustentable que mejora el ambiente debe estar basada en principios agroecológicos y en un método participativo en el desarrollo y difusión de tecnología. La agroecología es la ciencia que se basa en los principios ecológicos para el diseño y manejo de sistemas agrícolas en cuanto a conservación de recursos, y que ofrece muchas ventajas para el desarrollo de tecnologías más favorables para el agricultor. La agroecología se erige sobre el conocimiento indígena y tecnologías modernas selectas de bajos insumos para diversificar la producción. El sistema incorpora principios biológicos y los recursos locales para el manejo de los sistemas agrícolas, proporcionando a los pequeños agricultores una forma ambientalmente sólida y rentable de intensificar la producción en áreas marginales. 
Para que beneficie a los campesinos pobres, la investigación y el desarrollo agrícolas deberían operar sobre la base de un enfoque "de abajo hacia arriba", usando y construyendo sobre los recursos disponibles (la población local, sus conocimientos y sus bienes naturales nativos). Deben tomarse muy en serio las necesidades, aspiraciones y circunstancias particulares de los pequeños agricultores, mediante métodos participativos. Esto significa que desde la perspectiva de los agricultores pobres, las innovaciones tecnológicas deben:

- Ahorrar insumos y reducir costos.

- Eliminar riesgos.

- Expandirse hacia tierras marginales frágiles.

- Ser congruentes con los sistemas agrícolas campesinos.

- Mejorar la nutrición, salud y ambiente.

Precisamente, debido a estos requerimientos la agroecología ofrece más ventajas que la Revolución Verde y los métodos biotecnológicos. Las características promisorias de las técnicas agroecológicas son los siguientes:
- Se basan en el conocimiento indígena y la racionalidad campesina

- Son económicamente viables, accesibles y basadas en recursos locales

- Son sanas para el ambiente y sensibles desde el punto de vista social y cultural

- Evitan el riesgo y se adaptan a las condiciones del agricultor

- Mejoran la estabilidad y productividad de la finca y no sólo de cultivos.

Hay miles de casos de productores rurales que, en asociación con ONGs y otras organizaciones, promueven sistemas agrícolas y conservan los bienes manteniendo altos rendimientos y cumpliendo con los criterios antes mencionados. Aumentos de 50 a $100 \%$ en la producción son comunes con la mayoría de estos métodos de producción.

\section{Resumen}

La tendencia hacia una visión reduccionista del agroecosistema, promovida por la biotecnología contemporánea, debe ser revertida por un enfoque holístico, para asegurar que las alternativas agroeco- 
lógicas no sean ignoradas y que sólo se investiguen y desarrollen aspectos biotecnológicos ecológicamente aceptables. Es necesario enfrentar efectivamente el reto y realidad de la ingeniería genética. Como ha ocurrido con los plaguicidas, las compañías de biotecnología deben sentir el impacto de los movimientos ambientalistas, laborales y campesinos, de modo que reorienten su trabajo para beneficio de toda la sociedad y naturaleza. El futuro de la investigación agrobiotecnológica estará determinado por relaciones de poder y no hay razón para que los agricultores y el público en general, si se organizan y logran suficiente poder, no puedan influir en la dirección de la biotecnología, de modo que cumpla con las metas de la sustentabilidad.

La encrucijada social y ecológica que enfrenta la sociedad requiere que el científico, informado por los conceptos de desarrollo sustentable y principio precautorio, cuestione e indague quién dirige el desarrollo de la ciencia, quién la financia, respondiendo a qué intereses y con cuáles propósitos se realizan.

La controversia que surge es que siempre están los detractores de todo cambio y toda tecnología. Y siempre hay que aceptar que cuando viene una nueva tecnología, como es nueva, uno no sabe los efectos secundarios o dañinos que pueda tener.

Se puede observar entonces que uno de los problemas que ha desencadenado el debilitamiento del ecosistema, es la ausencia de aspectos morales a la hora de llevar a la práctica los diferentes conocimientos científico-tecnológicos, a sabiendas de que nuestro deber como seres humanos y principales administradores de los recursos que nos ofrece la biosfera es hacer buen uso de estos, considerando además que también sabemos que la naturaleza es un sistema vivo y se verá afectado por el abuso y mal manejo que se le dé.

Es en estas circunstancias tan adversas para la humanidad y para toda la vida en la tierra, que surge la bioética (desde el punto de vista ambiental) como herramienta para un mayor control y supervisión de las actividades que realiza el ser humano y las repercusiones que tendrán éstas sobre la naturaleza en este caso. Es indispensable la reciente de la ética ambiental como instrumento impulsor de una conciencia ambiental y social 
responsable y activa, promovedora de una mentalidad crítica del ser humano que conduzca al desarrollo de actitudes y aptitudes concretas ante las situaciones que se consideren negativas para la naturaleza y que además pueda ser transmitida a las futuras generaciones para una mejor calidad de vida humana y de las demás especies existentes.

El modelo agroindustrial se agota, no es la solución, como pretenden los gobiernos (político-empresarios), sino que es la causa de los problemas socio-ambientales actuales. Por eso, se debe replantear todo el sistema de dominación convencional (instituciones, leyes), que como modelo está en franca decadencia e implantar uno que rompa con las estructuras nacionales e internacionales, acorde con la necesidad de los tiempos.

Sin embargo, para generar un proceso de estructuras políticas socio-ambientales es necesario cuestionar el desarrollo convencional con cambios estructurales (institucionales, legislativos) en los que el ciclo económico respete el ciclo ecológico. Estos cambios deben conllevar a una relación socio-económica y ambiental más justa y equitativa que beneficie a la mayoría y no solo a una minoría (Toledo, 1990).
La sociedad debe asumir un desarrollo sustentable, basado en los principios de equidad social, respeto a la integridad ecológica de los ecosistemas, modelo económico alternativo y democracia participativa.

Se trata de tomar partido por una opción viable para el futuro de los seres humanos y de la naturaleza. La concepción sustentable y su relación con su entorno es fundamentalmente una opción de vida, una opción humana que la agroética, como alternativa de desarrollo sustentable, nos puede proporcionar realmente.

\section{Bibliografía}

Altieri MA. (1999), Alternatives to conventional modern agriculture for meeting world food needs in the next century. Report of a Bellagio Conference. Ithaca, NY: Cornell International Institute for Food, Agriculture and Development.

Altieri, M.A. (2000), Biotecnología agrícola: mitos, riesgos ambientales y alternativas. Universidad de California, Berkeley. PED-CLADES / FOOD FIRST.

Altieri, M. y Nicholls, C. (2000), Agroecología: teoría y práctica para una agricultura sostenible. Serie Textos Básicos para la Formación Ambiental. ONU-PNUMA. 
Altieri MA, Rosset P, Thrupp LA. (1998), The potential of agroecology to combat hunger in the developing world. (2020 Brief $\mathrm{N}^{\circ}$ 55). Washington DC: International Food Policy Research Institute.

Aristide JB. (2000), Eyes of the heart: seeking a path for the poor in the age of globalization. Monroe ME: Common Courage Press.

Beck, U. (1998), La sociedad de riesgos: hacia una nueva modernidad. Barcelona, Paidós.

Blázquez, Niceto (2000), Bioética. La nueva ciencia de la vida. Madrid: BAC. Busch L, Lacy WB.

Burkhardt J, et al. (1990), Plants, Power and Profit. Brasil Blackwell. Editorial Oxford:

Carvajal, A. (2003), La Bioética: conceptos y características. Revista Girasol. Vol.:5, $198 \mathrm{p}$.

Heller, A. (1995), Ética general. Madrid. Centro de Estudios constitucionales. Elliot, Robert () La Ética Ambiental.

FAO (2002), Seguridad Alimentaría Mundial y Plan de Acción Mundial sobre la Alimentación. Roma, Editorial supongo FAO.

Fowler C, Mooney P. (1990), Shattering: Food, Politics and the Loss of Genetic Diversity. Tucson: University of Arizona Press.

Gafo, J. (2001), 10 palabras claves en bioética. España. 385 p. Editorial Verbo Divino.

García, Jaime (2004), 30 razones contra los cultivos transgénicos en Costa Rica. Ambien-tico. Núm. 132, sep, 2004. Escuela de Ciencias Ambientales de la Universidad Nacional, Heredia, Costa Rica.
Garrido Peña, Francisco (2002), Teoría de la elección racional y biotecnología de transgénicos. En Benítez Ortúzar, Ignacio F. (coord.). Genética humana en el tercer milenio. Aspectos éticos y jurídicos. Madrid: Ediciones AKAL, 2002. Universidad Internacional de Andalucía.

Hindmarsh R. (1991), The flawed "sustaina$b l e^{\prime \prime}$ promise of genetic engineering. The Ecologist 1991; 21: 196-205.

Jonas, Hans (1995), El principio de responsabilidad. Barcelona: Editorial Herder.

Kieffer, G. (1983), Bioética. Madrid. Editorial Alambra, 495 p.

Krimsky S, Wrubel RP. (1996), Agricultural Biotechnology and the Environment: Science, Policy and Social Issues. Urbana: University of Illinois Press.

Lappé FM, Collins J, Rosset P. (1998), World hunger: twelve myths. New York: Editorial Grove Press.

Leff, E. (1990), La ética del ecodesarrollo: hacia una racionalidad ambiental. Revista de la Universidad Autónoma de Yucatán.

Lolas, F. (2000), Bioética y Antropología médica. Chile. Editorial Mediterráneo. $174 \mathrm{p}$.

May, Roy. (2002), Ética y medio ambiente: hacia una vida sostenible. San José: Editorial DEI.

Manifiesto por la Vida. (2002), Por una Ética para la Sustentabilidad Simposio sobre Ética y Desarrollo Sustentable, Bogotá, 2-4 mayo.

Mansalve Medina, Luisa. (2000), Ética y bioética: un ensayo de aproximación. Franciscanum. Revista de Ciencias del Espíritu. Núm. 126, sep-dic. 
Marlasca, A. (2001), Introducción a la bioética. EUNA, Costa Rica. 286 p.

Meeroff, Marcos (1997), Ética y ecología. Palabra y Persona. Vol. 1, núm. 1, 1997

Costa, Mario L. (1998) Cómo hacer cosas con criterios y principios. Anamnesis.Núm. 16, jul-dic.

Provencio, Enrique y Saucedo, Beatriz (1999) Ética y medio ambiente. Umbral XXI, núm. 30. Restrepo Mejía,

Luz M. (2000), De la ética a la bioética. Revista Universidad deAntioquia. Núm. 259, ene-mar, Medellín Colombia

Rosset P. (1999), The Multiple Functions and Benefits of Small Farm Agriculture in the Context of Global Trade Negotiations. (Food First Policy Brief $\left.N^{\circ} 4\right)$. Oakland, CA: Institute for Food and Development Policy.

Sachs, W (ed) (1998), The Development Dictionary: A Guide to Knowledge as Power. Bolivia: AGRUCO.

Sgreccia, E. (1996), Manual de Bioética, México. 688 p. Editorial Diana. Singer, Peter. Compendio de Ética.

Snow AA, Moran P. (1997), Commercialization of transgenic plants: potential ecological risks. BioScience 1997; 47: 86-96.

Toledo, V.M. (1990), Modernidad y Ecología: la nueva crisis planetaria. En Ecología Política nำ; pp.9-22.

Trevijano, M. (1999), ¿Qué es la bioética? España. 263 p. Editorial Sígueme. Uphoff $\mathrm{N}$. 